\title{
Contribuição da Tomografia Computadorizada em Pacientes com Metástases Pulmonares de Carcinoma Diferenciado de Tireóide Não-Aparentes na Radiografia e Tratados com Radioiodo
}

\section{artigo original}

\author{
PEDRO W. S. ROSÁRIO \\ WILSON C. TAVARES \\ ÁlVARO L. BARROSO \\ LEONARDO L. REZENDE \\ EDUARDo L. PADRÃo \\ Saulo Purisch
}

Departamento de Tireóide, Serviço de Endocrinologia, Santa

Casa Belo Horizonte, MG, Brasil

(PWSR, SP); Instituto Alpha de Gastroenterologia, Belo Horizonte, MG (WCT); Serviço de Medicina

Nuclear, Santa Casa Belo Horizonte, MG, Brasil $(A L B, L L R, E L P)$

Recebido em 01/02/2007 Aceito em 06/07/2007

\section{RESUMO}

A tomografia computadorizada (TC) de tórax é mais sensível que a radiografia na detecção de metástases pulmonares do carcinoma diferenciado de tireóide (CDT), sendo importante conhecer melhor o valor agregado desse método. Este estudo avaliou a resposta ao tratamento com ${ }^{131} \mathrm{I}$ em pacientes com metástases pulmonares de CDTs não-aparentes na radiografia e o valor da TC nesses casos. Foram avaliados 25 pacientes com metástases pulmonares não-aparentes na radiografia, que receberam inicialmente 100 a 200 $\mathrm{mCi}$ de ${ }^{131} \mathrm{I}$. Naqueles com pesquisa de corpo inteiro ( $\mathrm{PCl}$ ) pós-dose com captação pulmonar, um novo tratamento era realizado após seis a 12 meses, e assim sucessivamente. A chance do encontro de captação pulmonar na $\mathrm{PCl}$ pós-dose não foi diferente em pacientes com TC negativa ou positiva $1100 \%$ versus $91,5 \%$ ). Os valores médios de tireoglobulina ( $\mathrm{Tg}$ ) sérica foram maiores naqueles com TC positiva (108 ng/mL versus $52 \mathrm{ng} / \mathrm{mL}$ ). $\mathrm{PCl}$ pós-dose negativa foi alcançada em $82 \%$ dos pacientes com TC positiva e em $92,3 \%$ com TC negativa, e a atividade acumulada de ${ }^{131} \mathrm{I}$ para alcançar essa resposta não foi diferente nos dois grupos (em média, $300 \mathrm{mCi}$ ). Quarenta e sete por cento dos pacientes com TC negativa ao final do tratamento apresentaram Tg estimulada indetectável, mas nenhum daqueles que permaneceu com TC positiva apresentou-a. Em pacientes com Tg elevada, o resultado da TC aparentemente não altera a indicação da terapia e a atividade de ${ }^{131}$ l a ser administrada. Nos casos com metástases pulmonares, a permanência de micronódulos na TC nos pacientes com PCl pós-dose negativa após o tratamento foi associada à persistência de Tg detectável. (Arq Bras Endocrinol Metab 2008;52/1:114-119)

Descritores: Tomografia computadorizada; Metástases pulmonares; Câncer de tireóide

\section{ABSTRACT}

Contribution of Computed Tomography in Patients with Lung Metastases of Differentiated Thyroid Carcinoma Not Apparent on Plain Radiography who Were Treated with Radioiodine.

Computed tomography (CT or CAT Scan) of the chest is more sensitive than radiography in the detection of lung metastases of differentiated thyroid cancer (DTC), but little information is available regarding the aggregated value of this method. The present study evaluated the response of patients with lung metastases of DTC not apparent on radiography to treatment with ${ }^{131} \mathrm{I}$ and the value of CT in these cases. Twenty-five patients with lung metastases not apparent on radiography, who initially received $100-200 \mathrm{mCi} \mathrm{I}{ }^{151}$, were evaluated and those presenting pulmonary uptake on post-therapy WBS were submitted to a new treatment after 6 to 12 months, and so on. The chance of detection of pulmonary uptake on post-therapy WBS did not differ between patients with negative and positive CT (100\% versus $91.5 \%)$. Mean serum $\mathrm{Tg}$ levels were higher in patients with positive CT (108 $\mathrm{ng} / \mathrm{ml}$ versus $52 \mathrm{ng} / \mathrm{ml})$. Negative post-therapy 
WBS was achieved in $82 \%$ of patients with positive CT and in $92.3 \%$ with negative CT and the cumulative I $^{131}$ activity necessary to achieve this outcome did not differ between the two groups (mean $=300 \mathrm{mCi}$ ). Stimulated Tg was undetectable in $47 \%$ of patients with negative CT at the end of treatment, but in none of the patients whose CT continued to be positive. In patients with elevated Tg, the CT result apparently did not change the indication of therapy or the $\mathrm{I}^{131}$ activity to be administered. In cases with lung metastases, the persistence of micronodules on CT was associated with the persistence of detectable $\mathrm{Tg}$ in patients presenting negative post-therapy WBS. (Arq Bras Endocrinol Metab 2008;52/1:114-119)

Keywords: Computed tomography; Lung metastases; Thyroid cancer

\section{INTRODUÇÃO}

U MA MINORIA DOS PACIENTES com câncer diferenciado de tireóide (CDT) apresenta metástases pulmonares, e a presença destas piora o prognóstico (1). Essas metástases são geralmente assintomáticas e tardiamente aparentes na radiografia $(2,3)$, sendo identificadas precocemente por meio da pesquisa de corpo inteiro com ${ }^{131} \mathrm{I}$ (PCI), especialmente após a administração de altas atividades a pacientes com Tg elevada (4), e eventualmente na tomografia computadorizada (TC) de tórax. A importância do diagnóstico precoce é claramente demonstrada por Schlumberger (1999) que relata sobrevida de $93 \% \mathrm{em} \mathrm{dez}$ anos quando a radiografia é normal, de 63\% quando esta revela micrometástases (lesões $\leq 1 \mathrm{~cm}$ ) e de apenas $11 \%$ quando macrometástases são encontradas (5).

A TC é mais sensível que a radiografia simples para detecção de metástases pulmonares $(2,4,6-8)$, sendo proposta como exame radiológico de primeira linha na suspeita desse acometimento (8). Em pacientes com $\mathrm{Tg}$ sérica elevada, sem doença aparente, a indicação de uma atividade empírica de ${ }^{131} \mathrm{I}$ muda se a TC for normal ou revelar micrometástases? A chance de as metástases serem iodocaptantes (por exemplo: aparentes na PCI pós-dose) é menor em pacientes com TC positiva que naqueles com TC negativa? A resposta à radioiodoterapia e o prognóstico destes últimos é melhor do que nos primeiros?

O objetivo deste estudo foi avaliar a resposta ao tratamento $\mathrm{com}^{131} \mathrm{I}$ em pacientes com metástases pulmonares assintomáticas e não aparentes na radiografia, portanto precocemente diagnosticadas, e o valor agregado da TC nesses casos.

\section{PACIENTES E MÉTODOS}

Todos os pacientes com CDT que receberam uma atividade de ${ }^{131}$ I para ablação de remanescentes tireoidianos (ART) ou tratamento de metástases em nossa instituição (Santa Casa de Belo Horizonte, Brasil) a partir de 2001 foram avaliados com exame clínico, radiografia de tórax, dosagem de $\mathrm{Tg}$ sérica e anticorpos anti-Tg (AATg) imediatamente antes e com uma PCI sete dias após a administração do ${ }^{131} \mathrm{I}$, independentemente dos exames prévios. Com base nos resultados dessa avaliação de rotina, a investigação era ampliada. TC de tórax foi realizada em pacientes com captação pulmonar na PCI pós-dose; com $\mathrm{Tg}>5 \mathrm{ng} / \mathrm{mL}$ e AATg negativos; ou com AATg presentes e PCI pós-dose negativa. Ultra-sonografia (US) cervical foi realizada em pacientes com exame clínico normal e captação de ${ }^{131} \mathrm{I} \mathrm{em}$ linfonodos cervicais na PCI pós-dose e, associada à TC, naqueles com $\mathrm{Tg}>5 \mathrm{ng} / \mathrm{mL}$ e AATg negativos ou com AATg presentes e PCI pós-dose sem metástases. Outros métodos de imagem foram realizados apenas na presença de sintomas que sugerissem o acometimento de um sítio específico. Poucos pacientes tinham realizado uma PCI com baixa atividade de ${ }^{131} \mathrm{I}(4$ a $5 \mathrm{mCi}$ ) antes de serem encaminhados para radioiodoterapia. Nesses casos, o intervalo entre a administração da atividade traçadora e a terapêutica foi maior que 90 dias.

Para este estudo, foram selecionados 25 pacientes que atenderam aos seguintes critérios: submetidos à tireoidectomia total com dissecção cervical (nos casos com acometimento linfonodal aparente na cirurgia), assintomáticos no momento da avaliação, com exame clínico e radiografia de tórax normais, com metástases 
pulmonares detectadas na PCI pós-dose e/ou na TC de tórax, e sem metástases ósseas ou linfonodais aparentes durante a investigação anteriormente descrita. As características e o estadiamento iniciais desses pacientes, de acordo com a American Joint Committee on Cancer (AJCC/TNM) (9), encontram-se na tabela 1. Em 15 pacientes, a terapia com ${ }^{131} \mathrm{I}$ estava sendo realizada pela primeira vez após a tireoidectomia, com a intenção de promover ART. Dez pacientes já haviam recebido ${ }^{131} \mathrm{I}$ para ART, com PCI pós-dose não mostrando captação pulmonar, sendo encaminhados a nova terapia com ${ }^{131} \mathrm{I}$ por apresentarem $\mathrm{Tg}$ elevada $(\mathrm{n}=6)$ ou PCI com 5 $\mathrm{mCi}$ de ${ }^{131} \mathrm{I}$ positiva $(\mathrm{n}=4)$ durante o seguimento. Portanto, as metástases pulmonares eram conhecidas antes do estudo em apenas $4 / 25$ pacientes.

Os pacientes com metástases pulmonares aparentes na PCI pós-dose receberam 100 a $200 \mathrm{mCi}$ de ${ }^{131} \mathrm{I}$ por ciclo, em intervalo de 6 a 12 meses (média 8 meses), após suspensão de levotiroxina, sempre realizando dosagem de Tg sérica estimulada e AATg imediatamente antes e uma PCI sete dias após a administração do ${ }^{131} \mathrm{I}$. Nenhum paciente realizou PCI com baixa atividade de ${ }^{131} \mathrm{I}$ ( $5 \mathrm{mCi}$ ) nos intervalos entre as terapias. Quando a PCI pós-dose tornava-se negativa, o paciente era submetido à TC de tórax. Foi realizada a dosagem da Tg estimulada 12 a 18 meses após a PCI pós-dose negativa, intervalo definido com base na possibilidade de efeito tardio do ${ }^{131} \mathrm{I}$ em negativar os métodos de imagem $(2)$ e a $\operatorname{Tg}(2,10,11)$. O TSH foi mantido $\leq 0,1$ $\mathrm{mUI} / 1$ nos intervalos entre as terapias com ${ }^{131} \mathrm{I}$.

Este estudo foi aprovado pelo Comitê de Ética em Pesquisa da instituição.

\section{Dosagem da tireoglobulina sérica}

$\mathrm{Tg}$ foi determinada por ensaio imunorradiométrico (ELSA-hTG, CIS bio internacional, França) com sensibilidade funcional de $1,0 \mathrm{ng} / \mathrm{mL}$, precisão intra-ensaio de $6,6 \%$, $3,3 \%$ e $2,4 \%$ para valores de $4,9,100,8$ e $263,7 \mathrm{ng} / \mathrm{mL}$, respectivamente, e precisão inter-ensaio (intervalo de dois meses) de $8 \%, 6,9 \%$ e 5,1\% para valores de 4,9, 223,2 e 312,9 ng/mL, respectivamente. Seguindo a recomendação da NACB (National Academy of Clinical Biochemistry) (12), estabelecemos o valor de referência em nossa população normal eutireoidiana que foi de 3 a $42 \mathrm{ng} / \mathrm{mL}$. AATg foram medidos usando um ensaio quimiluminescente (Chemiluminescent ICMA, Nichols Institute Diagnostic, San Juan Capistrano, CA), com limite de detecção de 1,0 UI/mL, precisão intra e inter-ensaios de $8,7 \%$ e $5,9 \%$ para valores de 2 a 40 $\mathrm{UI} / \mathrm{mL}$ (valores fornecidos pelo fabricante) e valor de referência até $2 \mathrm{UI} / \mathrm{mL}$.

\section{Métodos de imagem}

Pesquisa de corpo inteiro foi realizada sete dias após a administração de 100 a $200 \mathrm{mCi}$, após suspensão de levotiroxina por até cinco semanas, alcançando valores de TSH superiores a $30 \mathrm{mUI} / \mathrm{L}$, e prescrição de dieta hipoiódica durante duas semanas antes do tratamento. Foram obtidas imagens anteriores e posteriores de corpo inteiro.

Outros métodos de imagens ocasionalmente usados para definir o estado da doença foram a radiografia simples de tórax, a ultra-sonografia cervical, a tomografia computadorizada (TC) de tórax e o mediastino sem contraste e com cortes seqüenciais de 5 a $10 \mathrm{~mm}$.

\section{Análise estatística}

A análise estatística foi realizada pelo software Minitab, por meio do teste do $\mathrm{X}^{2}$, considerando significante um valor de $\mathrm{p}<0,05$.

\section{RESULTADOS}

Em pacientes com metástases pulmonares e radiografia normal, a chance do encontro de captação pulmonar na PCI pós-dose não foi diferente em pacientes com TC negativa ou positiva $[13 / 13(100 \%)$ versus $11 / 12$ $(91,5 \%)]$. A idade na ocasião do diagnóstico das metástases, tipo histológico, e estadiamento do tumor (tamanho, invasão e metástases linfonodais) também não foram diferentes entre pacientes com TC positiva ou negativa; 20/25 pacientes apresentavam AATg negativos e todos estes tinham $\mathrm{Tg}$ estimulada $>10 \mathrm{ng} / \mathrm{mL}$, com valores médios maiores em pacientes com TC positiva ( $108 \mathrm{ng} / \mathrm{mL}$ versus $52 \mathrm{ng} / \mathrm{mL}(\mathrm{p}<0.05)$. (Tabela 1 , Figura 1)

PCI pós-dose negativa após o tratamento com ${ }^{131} \mathrm{I}$ foi obtida em $9 / 11(82 \%)$ pacientes com TC positiva e em 12/13 (92,3\%) com TC negativa, todos estes com a PCI pós-dose inicial positiva (Figura 1). A atividade acumulada de ${ }^{131}$ I para alcançar essa resposta (excluindo o último ciclo, em que a PCI pós-dose foi negativa) variou de 150 a $500 \mathrm{mCi}$ (média $300 \mathrm{mCi}$ ) e não foi diferente em pacientes com ou sem metástases aparentes na TC inicial. Os três pacientes que permanecer- 
Tabela 1. Características e estadiamento iniciais (9) dos pacientes avaliados neste estudo.

\begin{tabular}{|c|c|c|c|c|c|c|c|c|}
\hline Sexo & $\begin{array}{l}\text { Idade } \\
\text { (anos) }\end{array}$ & $\begin{array}{c}\text { Tipo } \\
\text { histológico }\end{array}$ & $\begin{array}{l}\text { Tamanho do } \\
\text { tumor }(\mathrm{cm})\end{array}$ & $\begin{array}{c}\text { Invasão } \\
\text { extratireoidiana* }\end{array}$ & $\begin{array}{l}\text { Metástases } \\
\text { linfonodais }\end{array}$ & $\underset{(\mathrm{Tg} / \mathrm{mL})^{* *}}{\left({ }^{*}\right.}$ & TC & $\mathrm{PCl}^{* * *}$ \\
\hline $\mathrm{F}$ & 13 & Papilífero & $1,7^{\star \star \star \star}$ & Ausente & NO & 102 & Positiva & Positiva \\
\hline M & 28 & Folicular & 5 & Ausente & NO & $\mathrm{AATg}+$ & Negativa & Positiva \\
\hline $\mathrm{F}$ & 39 & Papilífero & 1,5 & Ausente & N1 & 36 & Negativa & Positiva \\
\hline $\mathrm{F}$ & 16 & Papilífero & 4,2 & Ausente & N1 & 96 & Positiva & Positiva \\
\hline $\mathrm{F}$ & 51 & Papilífero & 3,5 & Invasão extensa (T4) & N1 & 212 & Positiva & Positiva \\
\hline $\mathrm{F}$ & 43 & Folicular & 4,5 & Ausente & NO & 78 & Positiva & Positiva \\
\hline M & 18 & Papilífero & 3 & Ausente & NO & 80 & Negativa & Positiva \\
\hline $\mathrm{F}$ & 16 & Folicular & 4,3 & Invasão mínima (Т3) & $\mathrm{N} 1$ & 168 & Positiva & Positiva \\
\hline $\mathrm{F}$ & 37 & Papilífero & 2,5 & Ausente & $\mathrm{N} 1$ & 26 & Negativa & Positiva \\
\hline M & 27 & Papilífero & 4,5 & Ausente & N1 & 112 & Negativa & Positiva \\
\hline M & 45 & Folicular & 4,5 & Invasão extensa (T4) & N1 & 32 & Negativa & Positiva \\
\hline $\mathrm{F}$ & 19 & Papilífero & 3,7 & Ausente & N1 & 46 & Negativa & Positiva \\
\hline M & 23 & Papilífero & 4,1 & Ausente & N1 & AATg + & Negativa & Positiva \\
\hline $\mathrm{F}$ & 28 & Papilífero & 2,7 & Invasão mínima (Т3) & $\mathrm{N} 1$ & 203 & Positiva & Positiva \\
\hline M & 39 & Papilífero & 2,2 & Ausente & N1 & 56 & Positiva & Positiva \\
\hline $\mathrm{F}$ & 44 & Folicular & 3 & Invasão mínima (Т3) & NO & AATg + & Negativa & Positiva \\
\hline $\mathrm{F}$ & 28 & Papilífero & 3.8 & Invasão extensa (T4) & N1 & 13 & Negativa & Negativa \\
\hline M & 36 & Folicular & 4,2 & Invasão mínima (Т3) & NO & 84 & Positiva & Positiva \\
\hline $\mathrm{F}$ & 27 & Folicular & 4,8 & Invasão mínima (T3) & N1 & 76 & Negativa & Positiva \\
\hline $\mathrm{F}$ & 46 & Papilífero & 2,5 & Invasão mínima (Т3) & N1 & 43 & Negativa & Positiva \\
\hline $\mathrm{F}$ & 52 & Papilífero & 2 & Invasão mínima (Т3) & $\mathrm{N} 1$ & 56 & Negativa & Positiva \\
\hline $\mathrm{F}$ & 28 & Papilífero & 2 & Invasão mínima (Т3) & N1 & 43 & Positiva & Positiva \\
\hline $\mathrm{F}$ & 30 & Folicular & 3,5 & Invasão mínima (T3) & N1 & AATg + & Positiva & Positiva \\
\hline M & 45 & Folicular & 3 & Invasão mínima (Т3) & NO & 78 & Positiva & Positiva \\
\hline M & 32 & Papilífero & 2,8 & Ausente & N1 & AATg + & Positiva & Positiva \\
\hline
\end{tabular}

* Invasão extratireoidiana mínima (T3): tecido peritireoidiano e músculo esternocleiodomastóide (9); ** Tg dosada imediatamente antes da administração do ${ }^{13}$ l;; ${ }^{* * *} \mathrm{PCl}$ pós-dose terapêutica; ${ }^{* * * *}$ Tumor multicêntrico, forma agressiva da variante folicular do carcinoma papilífero.

am com PCI pós-dose positiva receberam uma atividade acumulada de $600 \mathrm{mCi}$ (um) e $650 \mathrm{mCi}$ (dois). Esses pacientes não receberam novos ciclos de tratamento e permanecem sem evidência de progressão da doença.

Vinte de 21 pacientes com PCI pós-dose negativa tinham Tg estimulada dosada 12 a 18 meses após a última terapia $\mathrm{com}^{131} \mathrm{I}$, todos sem AATg. A Tg sérica foi indetectável em $8 / 20$ pacientes $(40 \%), 5 / 11$ com TC inicial negativa e $3 / 9 \mathrm{com}$ TC positiva pré-tratamento.
Valores de $\mathrm{Tg}<10 \mathrm{ng} / \mathrm{mL}$ foram obtidos em $72,7 \%$ dos casos com TC negativa inicialmente e em 55,5\% dos pacientes com TC inicial positiva. (Tabela 2)

Em relação a TC realizada após o tratamento, $47 \%$ dos pacientes com TC final negativa apresentaram $\mathrm{Tg}$ estimulada indetectável, mas nenhum daqueles que permaneceram com TC positiva ao final da terapia com ${ }^{131}$ I (Tabela 2). 


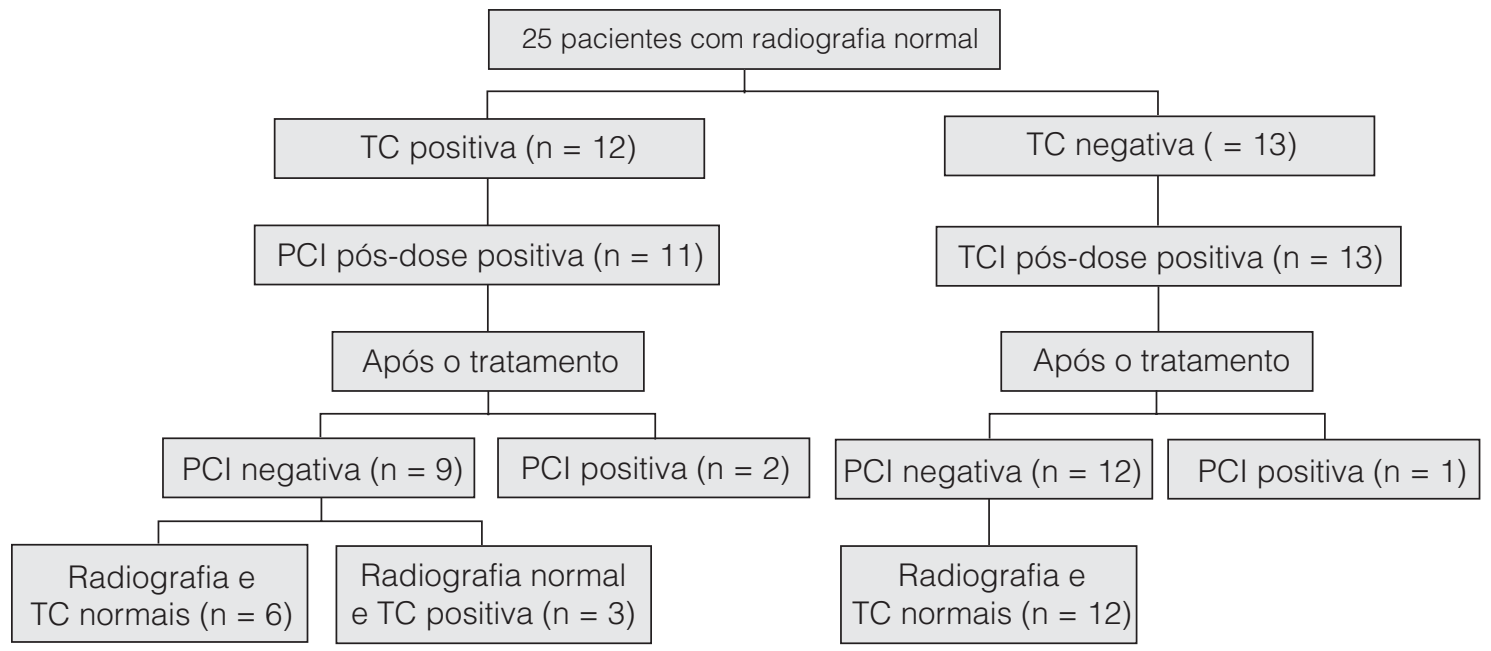

Figura 1. Resultados da TC e PCl pós-dose nos 25 pacientes com radiografia normal

Tabela 2. Valores de Tg estimulada nos pacientes com PCl pós-dose negativa e sem AATg

\begin{tabular}{lccc} 
Tg estimulada & Indetectável & $\mathbf{1}$ a $10 \mathbf{n g} / \mathbf{m L}$ & $>10 \mathrm{ng} / \mathbf{m L}$ \\
\hline TC negativa* & 8 & 6 & 3 \\
TC positiva* & 0 & 1 & 2 \\
\hline
\end{tabular}

* ao final do tratamento.

\section{DIsCUSSÃo}

Em pacientes com metástases pulmonares e radiografia normal, a TC mostrou micronódulos difusos em 50\% nessa série. Em outros estudos, esse número foi de $28 \%$ em crianças (7) e de aproximadamente $50 \%$ a $70 \%$ em adultos $(2,4,6,8)$. Nos casos com Tg elevada e PCI diagnóstica negativa, a TC é o método mais sensível para detecção de micrometástases pulmonares, sendo superior ao FDG-PET (13). Uma limitação da TC é a detecção de micronódulos periilares, pois estes não são facilmente diferenciados dos vasos dessa região (6).

Em pacientes com Tg elevada, a chance das metástases pulmonares (quando presentes) serem iodocaptantes (aparentes na PCI pós-dose) é elevada, 95\% nesta e em outra série (2), existindo indicação de uma atividade terapêutica empírica de ${ }^{131} \mathrm{I}$. A atividade de ${ }^{131} \mathrm{I}$ não necessariamente precisa ser maior que $100 \mathrm{mCi}$ naqueles com doença pulmonar aparente apenas na TC $(2,11)$.

Os níveis de $\mathrm{Tg}$ foram maiores em pacientes com TC positiva, concordante com estudos que mostram correlação da $\mathrm{Tg}$ sérica com a extensão da doença (14-16) e mais especificamente com o número de micronódulos vistos na TC (6).

Sabidamente, pacientes com metástases pulmonares não-aparentes na radiografia têm grande chance de resposta à radioiodoterapia $(2,11) ; 88 \%$ em nossa experiência com uma atividade de $300 \mathrm{mCi}$ (média), semelhante ao encontrado em outra série da literatura (2). O limite de $600 \mathrm{mCi}$ nos parece bem estabelecido, a partir do qual a chance de resposta é mínima e os efeitos adversos aumentam significativamente $(1,2,11)$, com risco considerável de neoplasia secundária (17). Como demonstrado neste estudo, a probabilidade de resposta é alta, e a radioiodoterapia promove o desaparecimento dos micronódulos em muitos casos $(2,4)$.

Após a negativação da PCI, como conseqüência da terapia com ${ }^{131} \mathrm{I}$, o achado de micronódulos na TC não é indicativo da necessidade de novos ciclos de tratamento (2,11). A evolução dessas lesões determinará a indicação ou não de outras modalidades terapêuticas.

Neste e em outros estudos $(6,7,8,11)$, o número de pacientes que negativou a PCI pós-dose e que apresentou micronódulos na TC foi pequeno para podermos comparar com aqueles com TC negativa ao final do tratamento. Apesar da possibilidade de micronódulos representarem apenas fibrose (6), o fato de os pacientes permanecerem com $\mathrm{Tg}$ detectável favorece a hipótese de doença persistente (6), mas não necessariamente implica pior prognóstico (11). Schlumberger mostra que pacientes que negativaram a PCI pós-dose permanecendo 
com Tg sérica detectável apresentaram maior mortalidade que aqueles com $\mathrm{Tg}$ indetectável (18). Como a persistência de micronódulos na TC foi associada aos níveis indetectáveis de Tg (TC positiva: Tg detectável em 100\% e TC negativa: Tg indetectável em quase 50\%), o achado de uma TC positiva após o tratamento pode estar relacionado com pior prognóstico.

\section{CONCLUSÕES}

Em pacientes com radiografia de tórax normal e Tg elevada, o resultado da TC de tórax não altera a indicação da terapia empírica e a atividade de ${ }^{131} \mathrm{I}$ a ser administrada. Nos casos com metástases pulmonares, a TC também não muda a conduta dos pacientes nos quais a PCI pós-dose torna-se negativa após o tratamento, mas a permanência de micronódulos na TC foi associada à persistência de $\mathrm{Tg}$ detectável.

\section{REFERÊNCIAS}

1. Schlumberger MJ. Papillary and follicular thyroid carcinoma. N Engl J Med. 1998;338:297-306.

2. Schlumberger M, Challeton C, De Vathaire F, Travagli JP, Gar$\operatorname{det} P$, Lumbroso JD, et al. Radioactive iodine treatment and external radiotherapy for lung and bone metastases from thyroid carcinoma. J Nucl Med. 1996;37:598-605.

3. Rosario PW, Barroso AL, Padrao EL, Rezende LL, Cardoso LD, Purisch S. Clinical manifestations and diagnosis of distant metastases of differentiated thyroid carcinoma after initial therapy. Arq Bras Endocrinol Metabol. 2004;48:861-6.

4. Schlumberger M, Arcangioli O, Piekarski JD, Tubiana M, Parmentier $\mathrm{C}$. Detection and treatment of lung metastases of differentiated thyroid carcinoma in patients with normal chest X-rays. J Nucl Med. 1988;29:1790-4.

5. Schlumberger MJ. Diagnostic follow-up of well-differentiated thyroid carcinoma: historical perspective and current status. J Endocrinol Invest. 1999;22(Suppl 11):3-7.

6. Piekarski JD, Schlumberger M, Leclere J, Couanet D, Masselot J, Parmentier C. Chest computed tomography (CT) in patients with micronodular lung metastases of differentiated thyroid carcinoma. Int J Radiat Oncol Biol Phys. 1985;11:1023-7.

7. Bal CS, Kumar A, Chandra P, Dwivedi SN, Mukhopadhyaya S. Is chest $\mathrm{x}$-ray or high-resolution computed tomography scan of the chest sufficient investigation to detect pulmonary metastasis in pediatric differentiated thyroid cancer? Thyroid. 2004; $14: 217-25$.
8. Ilgan S, Karacalioglu AO, Pabuscu Y, Atac GK, Arslan N, Ozturk $\mathrm{E}$, et al. lodine-131 treatment and high-resolution CT: results in patients with lung metastases from differentiated thyroid carcinoma. Eur J Nucl Med Mol Imaging. 2004;31:825-30.

9. American Joint Committee on Cancer Thyroid 2002. In: Greene FL, Page BL, Fleming ID, Fritz A, Balch CM, Haller BG, et al, (eds.). AJCC cancer staging handbook. 6ed. Chapter 8. New York: Springer, 2002. p. 89-98.

10. Baudin E, Do Cao C, Cailleux AF, Leboulleux S, Travagli JP, Schlumberger M. Positive predictive value of serum thyroglobulin levels, measured during the first year of follow-up after thyroid hormone withdrawal, in thyroid cancer patients. J Clin Endocrinol Metab. 2003;88:1107-11.

11. Durante C, Haddy N, Baudin E, Leboulleux S, Hartl D, Travagli $\mathrm{JP}$, et al. Long-term outcome of 444 patients with distant metastases from papillary and follicular thyroid carcinoma: benefits and limits of radioiodine therapy. J Clin Endocrinol Metab. 2006;91:2892-9.

12. Laboratory support for the diagnosis and monitoring of thyroid disease. National Academy of Clinical Biochemistry Professional Association. 2002. 125 pages. Disponível em: www.nacb.org.

13. Dietlein M, Scheidhauer K, Voth E, Theissen P, Schicha H. Fluorine-18 fluorodeoxyglucose positron emission tomography and iodine-131 whole-body scintigraphy in the follow-up of differentiated thyroid cancer. Eur J Nucl Med. 1997;24:1342-8.

14. Bachelot A, Cailleux AF, Klain M, Baudin E, Ricard M, Bellon N, et al. Relationship between tumor burden and serum thyroglobulin level in patients with papillary and follicular thyroid carcinoma. Thyroid. 2002;12:707-11.

15. Rosario PW, Guimaraes VC, Maia FF, Fagundes TA, Purisch S, Padrao EL, et al. Thyroglobulin before ablation and correlation with posttreatment scanning. Laryngoscope. 2005;115:264-7.

16. Schlumberger M, Baudin E. Serum thyroglobulin determination in the follow-up of patients with differentiated thyroid carcinoma. Eur J Endocrinol. 1998;138:249-52.

17. Rubino C, de Vathaire F, Dottorini ME, Hall P, Schvartz C, Couette JE, et al. Second primary malignancies in thyroid cancer patients. Br J Cancer. 2003;89:1638-44.

18. Schlumberger M. Therapy for advanced disease: conventional systemic therapies. In: Frontiers in Thyroid Cancer: Clinical Care and Research for the Future. Baltimore, USA, 2005.

\section{Endereço para correspondência:}

Pedro Weslley Souza Rosário

Centro de Estudos e Pesquisa da Clinica de Endocrinologia e Metabologia (CEPCEM)

Av. Francisco Sales, $1111,5^{\circ}$ andar, Ala D

30150-221 Belo Horizonte, MG

E-mail: pedrorosario@globo.com 